\title{
Qual é o custo da prescrição pelo nome de marca na judicialização do acesso aos medicamentos?
}

\author{
What is the cost of brand name prescriptions in the \\ judicialization of access to medicines?
}

\author{
Luís Fernando Nunes Alves Paim¹, Carine Raquel Batt², Gabriela Saccani², \\ Irene Clemes Küllkamp Guerreiro ${ }^{1,4}$
}

\begin{abstract}
Resumo
Objetivos: Estimar o custo e a economia da aquisição de medicamentos fornecidos por meio de ação judicial, considerando a aquisição de medicamentos de referência, genérico e similar. Métodos: Estudo descritivo e analítico das ações judiciais ( $n=186$ ) para acesso a medicamentos pleiteados na comarca de Antônio Prado/RS entre os anos de 2004 a 2015. Investigou-se o custo dos tratamentos em três cenários: A - a aquisição pela referência; B - a aquisição de genéricos; C - a aquisição do medicamento de menor valor. Resultados: O valor acumulado necessário para o cumprimento integral das ações judiciais ativas entre os anos de 2004 a 2015 seria de $R \$ 6.592 .936,58$ para o cenário $A$, de $R \$ 5.573 .571,19$ para o cenário $B$ e de $R \$ 5.357 .309,82$ para o cenário C. O percentual de economia comparativamente ao cenário A foi $13,44 \%$ pela aquisição de medicamentos genéricos (cenário B) e de 17,94\% pela aquisição do medicamento de menor valor (cenário C). Conclusões: As ações judiciais de acesso aos medicamentos deferidas pela marca de referência oneram o Sistema Único de Saúde (SUS) e ferem o princípio da livre concorrência que orienta as licitações públicas.
\end{abstract}

Palavras-chave: judicialização da saúde; assistência farmacêutica; farmacoeconomia; medicamentos.

\begin{abstract}
Aim: Estimate the cost and economics of the purchase of medicines provided through lawsuit considering the acquisition of branded medicines, generic and similar equivalent. Methods: The descriptive and analytical study of deferred lawsuits $(n=186)$ for access to medicines demanded in the Antonio Prado - RS, between the years 2004 to 2015, investigated the cost of the medicines for the full compliance of the shares considering three scenarios: A - the acquisition of brand name medicines; B - the acquisition of generic and $\mathrm{C}$ - acquisition of smaller value considering brand name drug, generic equivalent or similar. The financial impact of this difference was estimated as the annual cost of the acquisition of Pharmaceutical Services Basic Component (CBAF) of this municipality. Results: The accumulated amount required for full compliance with the active lawsuits between the years 2004 to 2015 would be $R \$ 6,592,936.58$ for scenario $A$, of $R \$ 5,573,571.19$ for the scenario $B$ and $R \$ 5,357,309.82$ for scenario C. The percentage saving compared to scenario A was $13.44 \%$ for the acquisition of generic drugs (scenario $B$ ) and $17.94 \%$ for the acquisition of lower drug (C scenario). This difference in cost of the branded drugs ordered through judicial processes is over than
\end{abstract}

1Programa de Pós-graduação em Farmacologia e Terapêutica, Instituto de Ciências Básicas da Saúde, Universidade Federal do Rio Grande do Sul (UFRGS) - Porto Alegre (RS), Brasil.

2Programa de Pós-graduação em Hepatologia, Departamento de Farmacociências, Universidade Federal de Ciências da Saúde de Porto Alegre (UFCSPA) - Porto Alegre (RS), Brasil.

${ }^{3}$ Faculdade de Farmácia, Universidade Federal do Rio Grande do Sul (UFRGS) - Porto Alegre (RS), Brasil.

4Departamento de Produção da Matéria Prima, Faculdade de Farmácia, Universidade Federal do Rio Grande do Sul (UFRGS) - Porto Alegre (RS), Brasil.

Trabalho realizado na Universidade Federal do Rio Grande do Sul (UFRGS) - Porto Alegre (RS), Brasil.

Endereço para correspondência: Irene Clemes Küllkamp Guerreiro - Departamento de Produção da Matéria Prima, Faculdade de Farmácia, Universidade Federal do Rio Grande do Sul (UFRGS), Av. Ipiranga, 2752 - Bairro Santana - CEP: 90610-000 - Porto Alegre (RS), Brasil - Email: irene@ufrgs.br

Fonte de financiamento: nenhuma.

Conflito de interesses: nada a declarar. 
6 years and budget means comparing scenario $B$ to $A$, and nearly 10 years comparing the scenario $C$ to $A$. Conclusions: The lawsuits of access to medicines that require brand name drugs further burden the public health system, and hurt the principle of free competition that guides public tenders, including the drug. As the Brazilian Sistema Único de Saúde (SUS) under the requirements shall be according to the Brazilian Common Denomination, the lawsuits for access to medicines should respect this guidance.

Keywords: health litigation; pharmaceutical services; pharmacoeconomics; medicines.

\section{INTRODUÇÃo}

A judicialização da saúde brasileira é um fenômeno multifatorial ${ }^{1}$ que prejudica a execução das políticas de saúde no âmbito do Sistema Único de Saúde (SUS), visto que o cumprimento das determinações judiciais para o fornecimento de medicamentos, insumos e serviços acarreta gastos elevados e não programados no orçamento da saúde ${ }^{2}$. Essa prática vem aumentando dramaticamente nos últimos anos ${ }^{3}$. Vários problemas têm sido relacionados à judicialização de medicamentos, como o requerimento de medicamentos experimentais, a pressão da indústria farmacêutica pela abertura de mercado e ainda a judicialização de medicamentos de alto custo para doenças raras ${ }^{4}$.

No Brasil, o direito à saúde é assegurado pela Constituição Federal (CF) promulgada no ano de $1988^{5}$ e regulamentado por meio da Lei ${ }^{\circ} 8.080 / 1990^{6}$. Apesar do aprimoramento e da organização dos serviços durante os últimos 30 anos, muitos brasileiros buscam a justiça para garantir o acesso aos seus tratamentos medicamentosos ou assegurar procedimentos cirúrgicos fundamentados na premissa de que a saúde é um dever do Estado ${ }^{3,4,7-9}$. Uma revisão dos estudos publicados sobre judicialização do acesso aos medicamentos encontrou que se trata de um fenômeno mundial, porém a maior parte dos estudos é brasileira, demonstrando a crescente importância desse fenômeno que envolve aspectos técnico-científicos, sociais e legais ${ }^{10}$.

Alguns autores destacam que o uso da via judicial é uma forma legítima de garantia do pleno acesso à assistência terapêutica individual $^{1}$, no entanto outros apontam que a via judicial desconsidera a escassez de recursos públicos da saúde, acrescido ao fato de que, mesmo que houvesse disponibilidade oportuna de recursos, a demanda sempre será maior que a oferta ${ }^{11}$.

Nos estudos que avaliam o fenômeno da judicialização de medicamentos no Brasil, muitos autores apontam para a solicitação de medicamentos padronizados em listas governamentais cujo acesso já estaria garantido ${ }^{12,13}$. Parte desse fenômeno pode ser explicada pelo desconhecimento das formas de acesso; já a outra parte, pela indisponibilidade do produto, pela diferença de forma farmacêutica e apresentação, e também pela preferência e definição de marca comercial do produto que deve ser fornecido.

Cabe destacar que no Brasil, conforme definido pela Lei $\mathrm{n}^{\circ}$ 9.787/1999 ${ }^{14}$, os medicamentos são classificados em Medicamento de Referência ou produto inovador, Medicamento Genérico e Medicamento Similar. Além disso, as prescrições e licitações no âmbito do SUS devem ser realizadas pela Denominação Comum
Brasileira (DCB), conforme a Lei $\mathrm{n}^{\circ} 8.666 / 1993^{15}$. A lei dos genéricos faz parte da política nacional de medicamentos que, entre outras diretrizes, busca ampliar o acesso aos medicamentos ${ }^{16,17}$. Diversos estudos demonstram as diferenças de preços entre as categorias, apontando uma expressiva redução de preço quando comparados os medicamentos genéricos ou similares aos de referência ${ }^{7,18-21}$. Apesar disso, muitos prescritores têm preferência por determinadas marcas e optam por prescrever pelo nome comercial, mesmo no âmbito do SUS $8,9,12,22,23$.

Considerando o crescimento exponencial das demandas judiciais para acesso a medicamentos no Brasil, associado à escassez de recursos na saúde (e que os medicamentos genéricos podem ser uma opção terapêutica intercambiável com os seus correspondentes de referência ou marca), este trabalho pioneiro tem por objetivo estimar o custo e a economia da aquisição de medicamentos fornecidos por meio de ação judicial considerando a aquisição de medicamentos de marca, genérico e similar equivalente.

\section{MÉTODOS}

O presente estudo é um trabalho descritivo realizado a partir da coleta de dados das ações judiciais deferidas para acesso a medicamentos pleiteados na comarca de Antônio Prado/RS entre os anos de 2004 a 2015. A coleta de dados foi conduzida a partir do sistema de gerenciamento de dispensação estadual Administração de Medicamentos (AME) e também do Sistema de Gestão Municipal (GEMUS). As variáveis pesquisadas foram o número de tratamentos judicializados, o medicamento demandado em cada tratamento e a sua quantidade a ser dispensada mensalmente.

Posteriormente, foram pesquisados os respectivos processos judiciais, dos quais se obtiveram o tipo de representação jurídica, o tipo de ação (contra o Estado do Rio Grande do Sul e/ou contra o município de Antônio Prado) e o respectivo número processual. Para tanto, foi utilizado o site do Tribunal de Justiça do Estado do Rio Grande do Sul, por meio da base de dados para consulta/acompanhamento processual ${ }^{24}$. Usou-se a busca por nome da parte autora. Todos os dados foram digitados em planilhas do Programa Microsoft Excel. O critério de inclusão dos processos judiciais neste trabalho foi ter uma ação judicial deferida contra o Estado do Rio Grande do Sul e/ou contra o município de Antônio Prado e ter pelo menos um medicamento pleiteado. Foram excluídos das ações os tratamentos que demandavam produtos para saúde e/ou correlatos. 
Os medicamentos que compuseram essas ações foram classificados segundo o Sistema Anatômico e Terapêutico Químico (Código ATC INDEX Principal) e foram avaliados quanto à presença nos diferentes componentes da assistência farmacêutica, usando-se, para tanto, a Relação Nacional Medicamentos Essenciais (RENAME) edição de 2014 ${ }^{25}$. A identificação do medicamento de referência para cada um dos fármacos foi a partir das listas A e B publicadas periodicamente pela Agência Nacional de Vigilância Sanitária (ANVISA) ${ }^{26}$.

O valor médio de cada unidade farmacológica foi calculado para cada uma das três opções terapêuticas atualmente disponíveis: referência, genérico e similar. A obtenção do preço unitário foi realizada a partir da planilha de preços máximos ao governo, edição de janeiro de 2014, utilizando-se embalagens não hospitalares, com ICMS de $17 \%$ para os medicamentos tributados e $0 \%$ para os medicamentos isentos por meio do convênio 087/2002. Para obtenção do valor unitário do medicamento de referência, foi utilizada a embalagem com o maior número disponível de unidades farmacêuticas, dividindo-se esse valor pelo número de formas farmacêuticas contidas. Para o cálculo do valor unitário dos medicamentos genéricos e similares, utilizou-se uma apresentação de cada laboratório produtor com o maior número disponível de formas farmacêuticas, dividindo-se esse valor pelo número de formas farmacêuticas nelas contidas. A partir destas, obteve-se o valor médio. Com isso, conseguiu-se o valor unitário médio de cada unidade farmacológica para cada um dos tratamentos para o ano de 2014.

Para permitir a comparabilidade dos preços unitários dos medicamentos nos anos estudados, empregou-se a variação anual do Índice Nacional de Preços ao Consumidor Amplo $(\text { IPCA })^{27}$, calculado pelo IBGE ${ }^{28}$. Para os anos de 2004 a 2013, o valor unitário de cada medicamento foi deflacionado em relação à média obtida para o ano de 2014 e inflacionado pelo mesmo índice para o ano de 2015. O uso desse indicador tomou por base a Lei no $10.742 / 2003$, que estabeleceu as normas de regulação do setor farmacêutico e definiu esse índice para fins de ajuste nos preços dos medicamentos no país ${ }^{29}$.

A estimativa do valor anual de cada tratamento foi mensurada pela multiplicação do número de unidades farmacêuticas pelo valor unitário da unidade farmacológica e pelo número de meses na qual esteve deferida, considerando os seguintes cenários:

- Cenário A: caso a administração pública fosse condenada a fornecer apenas o medicamento de referência para cada um dos tratamentos judicializados;

- Cenário B: caso as ações fossem deferidas pela DCB, permitindo a administração pública optar pela opção mais vantajosa entre o medicamento de referência e o seu correspondente genérico;

- Cenário C: caso as ações fossem deferidas pela DCB, permitindo a administração pública optar pela opção mais vantajosa entre o medicamento de referência, genérico ou similar intercambiável.

O valor médio do custo anual em Reais de cada ação judicial, independentemente do número de fármacos judicializados, foi mensurado pela divisão do número de ações deferidas em cada ano pelo valor estimado para os três cenários anteriormente apresentados. Ressalta-se que o número de ações foi corrigido para cada ano, excluindo-se o número de óbitos que eventualmente ocorreram.

Finalmente, para ilustrar a aplicação da economia gerada pelo cumprimento das ações desses três cenários, fez-se um comparativo com a aplicação desses recursos na execução do componente básico da assistência farmacêutica. Para tanto, foram consideradas a Portaria ${ }^{\circ} 1.555 / 2013^{30}$, que estabelece o valor de R $\$ 9,82$ por habitante/ano como piso de investimento nesse bloco de medicamentos, e a população do município no ano de 2010 de 12.833 habitantes.

A coleta de dados foi autorizada pela Secretaria Estadual de Saúde do Estado do Rio Grande do Sul e pela Secretaria Municipal de Saúde do município de Antônio Prado.

\section{RESULTADOS}

Entre os anos de 2004 a 2015, foram deferidas 186 ações judiciais contra o poder público para acesso a medicamentos no município de Antônio Prado. Em relação ao tipo de ação, $67,2 \%(\mathrm{~N}=125)$ mencionaram apenas o Estado como réu do processo; $2,2 \%(\mathrm{~N}=4)$ foram deferidas apenas contra o município; e 30,6\% (N=57), contra ambos os entes. O tipo de representação jurídica mostrou que $81,2 \%(\mathrm{~N}=151)$ das ações tiveram representação jurídica feita por advogados oriundos de escritórios particulares, $17,7 \%(\mathrm{~N}=33)$ foram demandadas pela Defensoria Pública, 0,5\% $(\mathrm{N}=1)$ foi movida pelo Ministério Público e 0,5\% (N=1) se tratava de uma Ação Civil Pública.

As 186 ações judiciais deferidas entre os anos de 2004 a 2015 determinaram o fornecimento de 781 medicamentos, dos quais 508 tratamentos foram demandados pelas 125 ações deferidas apenas contra o Estado, outros 28 tratamentos foram determinados pelas quatro ações movidas somente contra o município e 245 tratamentos compuseram as 57 ações demandadas contra ambos os entes.

Dos 781 tratamentos deferidos, 30,6\% ( $\mathrm{N}=239)$ estão atualmente disponíveis no componente básico da assistência farmacêutica, 7,6\% (N=59) são medicamentos disponibilizados pelo Estado do Rio Grande do Sul por meio do componente especial, $11,4 \%(\mathrm{~N}=89)$ pertencem ao componente especializado da assistência farmacêutica, $0,9 \%(\mathrm{~N}=7)$ congrega o componente estratégico da assistência farmacêutica e 49,6\% (N=387) não pertencem a nenhum componente e foram classificados como medicamentos fora das listas. 
Quanto à classificação ATC, os medicamentos destinados ao tratamento das enfermidades associadas ao sistema nervoso central responderam por $34,7 \%(\mathrm{~N}=271)$ do total, os medicamentos relacionados ao tratamento das doenças cardiovasculares compreenderam $28,4 \%(\mathrm{~N}=222)$, enquanto que aqueles destinados ao sistema respiratório congregaram $11,4 \%$ $(\mathrm{N}=89)$. Outras 10 classes farmacológicas também apareceram, entretanto apresentaram percentuais menos expressivos, os quais são apresentados na Tabela 1 .

Ao longo do período analisado, conforme a Tabela 2, observa-se um crescente aumento do número de ações judiciais para acesso a medicamentos: em 2004, havia quatro ações e, em 2015, 151, o que representa um aumento de mais de 37 vezes. Além disso, nota-se um aumento no custo médio da ação, independente do cenário de aquisição. Assim, o custo médio da ação aumentou pelo menos dez vezes, comparando o ano de 2015 com o de 2004.

A estimativa do valor em Reais necessário para o cumprimento das ações judiciais nos diferentes cenários é apresentada na Tabela 3. $\mathrm{O}$ valor estimado acumulado necessário para o cumprimento integral das ações judiciais entre os anos de 2004 a 2015 foi de $\mathrm{R} \$ 6.592 .936,58$ para o cenário $\mathrm{A}$, de $\mathrm{R} \$ 5.573 .571,19$ para o cenário B e de $\mathrm{R} \$ 5.357 .309,82$ para o cenário C. Os cenários B e $\mathrm{C}$, quando comparados ao cenário $\mathrm{A}$, apresentam um percentual de economia médio de 13,44 e 17,94\%, respectivamente.

Essa diferença de custo de aquisição dos medicamentos de marca solicitados de processos judicias representa o orçamento para a aquisição de medicamentos do componente básico da assistência farmacêutica de mais de seis anos, comparando o cenário $\mathrm{B}$ com $\mathrm{A}$, e de quase dez anos, comparando o cenário $\mathrm{C}$ com $\mathrm{A}$.

Tabela 1. Perfil das ações judiciais para acesso a medicamentos impetradas no município de Antônio Prado/RS entre os anos de 2004 a 2015 $(n=186)$

\begin{tabular}{|c|c|c|}
\hline Variável & $\mathbf{N}$ & $\%$ \\
\hline \multicolumn{3}{|l|}{ Esfera pública demandada $(\mathrm{n}=186)$} \\
\hline Somente contra o Estado & 125 & 67,2 \\
\hline Somente contra o município & 4 & 2,2 \\
\hline Contra ambos os entes & 57 & 30,6 \\
\hline \multicolumn{3}{|l|}{ Tipo de representação jurídica $(n=186)$} \\
\hline Escritório de advocacia privado & 151 & 81,2 \\
\hline Defensoria Pública & 33 & 17,7 \\
\hline Ministério Público & 1 & 0,5 \\
\hline Ação civil pública & 1 & 0,5 \\
\hline \multicolumn{3}{|c|}{ Tratamentos demandados $x$ esfera pública demandada $(n=781)$} \\
\hline Tratamentos demandados somente contra o Estado & 508 & 65,0 \\
\hline Tratamentos demandados somente contra o município & 28 & 3,6 \\
\hline Tratamentos demandado contra ambos & 245 & 31,4 \\
\hline \multicolumn{3}{|c|}{ Medicamento de acordo com as listas padronizadas no SUS $(n=781)$} \\
\hline Componente básico da assistência farmacêutica & 239 & 30,6 \\
\hline Medicamentos especiais (Programa do RS) & 59 & 7,6 \\
\hline Componente especializado da assistência farmacêutica & 89 & 11,4 \\
\hline Componente estratégico da assistência farmacêutica & 7 & 0,9 \\
\hline Nenhuma lista citada anteriormente & 387 & 49,6 \\
\hline \multicolumn{3}{|c|}{ Medicamentos de acordo com Classificação ATC INDEX Principal $(n=781)$} \\
\hline Agentes antineoplásicos e imunomoduladores & 11 & 1,4 \\
\hline Anti-infecciosos de uso sistêmico & 8 & 1,0 \\
\hline Dermatológicos & 4 & 0,5 \\
\hline Órgãos sensoriais & 17 & 2,2 \\
\hline Produtos antiparasitários & 3 & 0,4 \\
\hline Sangue e órgãos hematopoiéticos & 53 & 6,8 \\
\hline Sistema cardiovascular & 222 & 28,4 \\
\hline Sistema geniturinário e hormônios sexuais & 12 & 1,5 \\
\hline Sistema hormonal excluído insulina e hormônio sexuais & 13 & 1,7 \\
\hline Sistema musculoesquelético & 29 & 3,7 \\
\hline Sistema nervoso & 271 & 34,7 \\
\hline Sistema respiratório & 89 & 11,4 \\
\hline Trato alimentar e metabolismo & 49 & 6,3 \\
\hline
\end{tabular}


Tabela 2. Número e custo médio $(\mathrm{R} \$)$ das ações judiciais para medicamentos impetradas no município de Antônio Prado/RS entre os anos de 2004 a 2015 ( $n=186)$ de acordo com os cenários A, B e C

\begin{tabular}{ccccc} 
Ano & Número de ações & \multicolumn{3}{c}{ Custo médio anual da ação (R\$) } \\
\cline { 3 - 5 } 2004 & 4 & Cenário A & Cenário B** & Cenário C $^{* * *}$ \\
2006 & 15 & 825,17 & 620,37 & 575,10 \\
2005 & 23 & $2.949,14$ & $2.622,12$ & $2.568,59$ \\
2007 & 32 & $7.078,16$ & $6.528,01$ & $6.444,22$ \\
2008 & 37 & $5.907,30$ & $5.222,01$ & $5.138,54$ \\
2009 & 56 & $6.075,03$ & $5.227,44$ & $5.180,54$ \\
2010 & 80 & $5.122,07$ & $4.323,92$ & $4.238,71$ \\
2011 & 94 & $5.946,19$ & $5.148,05$ & $4.665,62$ \\
2012 & 109 & $7.946,01$ & $7.058,13$ & $6.432,55$ \\
2013 & 134 & $8.247,31$ & $7.199,40$ & $6.679,04$ \\
2014 & 149 & $7.768,67$ & $6.708,87$ & $6.216,27$ \\
2015 & 151 & $8.009,81$ & $6.867,36$ & $6.349,89$ \\
\hline
\end{tabular}

${ }^{*}$ Cenário A: caso a administração pública fosse condenada a fornecer apenas o medicamento de referência para cada um dos tratamentos judicializados; ${ }^{* *}$ Cenário B: caso as ações fossem deferidas pela Denominação Comum Brasileira, permitindo a administração pública optar pela opção mais vantajosa entre o medicamento de referência e seu correspondente genérico; ${ }^{* *}$ Cenário C: caso as ações fossem deferidas pela Denominação Comum Brasileira, permitindo a administração pública optar pela opção mais vantajosa entre o medicamento de referência, genérico ou similar intercambiável

Tabela 3. Estimativa dos valores anuais em Reais $(R \$)$ e por ano para o cumprimento das ações judiciais para medicamentos impetradas no município de Antônio Prado/RS entre os anos de 2004 a 2015 ( $n=186)$ de acordo com os cenários A, B e C

\begin{tabular}{|c|c|c|c|c|c|}
\hline \multirow[b]{2}{*}{ Ano } & \multicolumn{3}{|c|}{ Custo anual de aquisição da totalidade das ações judiciais (R\$) } & \multicolumn{2}{|c|}{ Economia comparativa (\%) } \\
\hline & Cenário $A^{*}$ & Cenário $\mathrm{B}^{\star *}$ & Cenário $C^{* * *}$ & $\begin{array}{l}\text { Cenário } \mathrm{B} \text { em } \\
\text { relação com } \mathrm{A}\end{array}$ & $\begin{array}{l}\text { Cenário } \mathrm{C} \text { em } \\
\text { relação com } \mathrm{A}\end{array}$ \\
\hline 2004 & $3.300,69$ & $2.481,48$ & $2.300,40$ & 24,82 & 30,31 \\
\hline 2006 & $44.237,16$ & $39.331,74$ & $38.528,83$ & 11,09 & 12,90 \\
\hline 2005 & $162.797,66$ & $150.144,22$ & $148.217,01$ & 7,77 & 8,96 \\
\hline 2007 & $189.033,54$ & $167.104,27$ & $164.433,43$ & 11,60 & 13,01 \\
\hline 2008 & $224.775,93$ & $193.415,31$ & $191.679,95$ & 13,95 & 14,72 \\
\hline 2009 & $286.836,15$ & $242.139,32$ & $237.367,55$ & 15,58 & 17,25 \\
\hline 2010 & $475.695,23$ & $411.844,22$ & $373.249,29$ & 13,42 & 21,54 \\
\hline 2011 & $746.924,96$ & $663.464,19$ & $604.659,27$ & 11,17 & 19,05 \\
\hline 2012 & $898.956,78$ & $784.735,12$ & $728.015,52$ & 12,71 & 19,02 \\
\hline 2013 & $1.041 .001,19$ & $898.988,52$ & $832.980,34$ & 13,64 & 19,98 \\
\hline 2014 & $1.193 .461,39$ & $1.023 .236,11$ & $946.134,31$ & 14,26 & 20,72 \\
\hline 2015 & $1.325 .915,90$ & $1.176 .686,69$ & $1.089 .743,92$ & 11,25 & 17,81 \\
\hline Soma/Média & $6.592 .936,58$ & $5.753 .571,19$ & $5.357 .309,82$ & 13,44 & 17,94 \\
\hline
\end{tabular}

*Cenário A: caso a administração pública fosse condenada a fornecer apenas o medicamento de referência para cada um dos tratamentos judicializados; ${ }^{* *}$ Cenário B: caso as ações fossem deferidas pela Denominação Comum Brasileira, permitindo a administração pública optar pela opção mais vantajosa entre o medicamento de referência e seu correspondente genérico; ${ }^{* *}$ Cenário C: caso as ações fossem deferidas pela Denominação Comum Brasileira, permitindo a administração pública optar pela opção mais vantajosa entre o medicamento de referência, genérico ou similar intercambiável

\section{DISCUSSÃO}

Muitos estudos têm relacionado a judicialização de medicamentos com as desigualdades no acesso à saúde $\mathrm{e}^{1,2,10,11}$. Contudo, considerando os resultados produzidos neste estudo, quando se olha para o tipo de representação jurídica, 81,2\% $(\mathrm{N}=151)$ das ações foram realizadas por advogados oriundos de escritórios particulares, enquanto que $17,7 \%(\mathrm{~N}=33)$ foram demandadas pela Defensoria Pública, $0,5 \%(\mathrm{~N}=1)$ foi movida pelo Ministério Público e 0,5\% (N=1) tratava-se de uma Ação Civil Pública. Esses resultados sugerem que os pacientes que recorrem às ações judiciais têm melhores condições financeiras e têm maior conhecimento dos direitos sociais do que aqueles que não o fazem. A primeira condição é confirmada pelo fato que esses pacientes podem arcar com as despesas processuais $\mathrm{e}$ a segunda pode estar relacionada a um maior grau de instrução desses autores em relação à população geral. Outro problema relacionado à judicialização descrito na literatura ${ }^{9}$ refere-se ao caráter individual dessas ações, visto que não se estendem a outras pessoas que possivelmente possam estar na mesma situação e que ingressaram com uma ação judicial, fato que incorre contra o princípio da universalidade que compõe o SUS. 
Quase metade dos tratamentos demandados judicialmente 49,6\% ( $\mathrm{N}=387)$ - é de fármacos que não pertencem a nenhuma lista de medicamentos padronizada pelo SUS. Resultados semelhantes foram descritos por outros trabalhos conduzidos no Brasil ${ }^{2,9,12,23}$. A solicitação de medicamentos fora de listas pode não representar ganhos terapêuticos reais, além de aumentar os riscos de aparecimento de eventos adversos inesperados? O produto da seleção de medicamentos é uma lista daqueles considerados essenciais para atender às necessidades sanitárias locais'. O grande dilema do acesso aos medicamentos é definir o que é necessidade e quais devem ser atendidas ${ }^{31}$, uma vez que o conceito de saúde não está unicamente relacionado à ausência da doença ${ }^{11}$, e sim a um conceito mais amplo, no qual o fornecimento sozinho de medicamentos é incapaz de alcançar melhores indicadores de saúde.

Em relação aos medicamentos judicializados, cujo acesso está definido por algum dos componentes da assistência farmacêutica, os especiais (programa existente apenas no Rio Grande do Sul, com o Estado responsável pelo financiamento) responderam por 7,6\% $(\mathrm{N}=59)$ dos pedidos, os pertencentes ao componente especializado da assistência farmacêutica somaram $11,4 \%(\mathrm{~N}=89)$, enquanto que os medicamentos do componente estratégico da assistência farmacêutica representaram apenas $0,9 \%(\mathrm{~N}=7)$ do total. Pode-se inferir que esses tratamentos podem ter sido judicializados por diversas causas: o usuário não procurou a rede pública previamente para a abertura de um expediente administrativo; o usuário não cumpriu os critérios de inclusão ou os trâmites elencados pelo respectivo Protocolo Clínico e Diretriz Terapêutica do Ministério da Saúde ou pela portaria equivalente; o usuário cumpriu os trâmites, teve o expediente administrativo deferido, entretanto não recebeu adequada e ininterruptamente o tratamento em virtude de eventuais falhas no abastecimento.

Quanto ao número expressivo - 30,6\% ( $\mathrm{N}=239)$ - de medicamentos solicitados via judicial, mas que estão padronizados no elenco do componente básico da assistência farmacêutica, há pelo menos duas explicações possíveis: o usuário não procurou previamente a rede municipal de farmácias ou o medicamento encontrava-se constantemente em falta nesses pontos de acesso. Para o aumento da precisão dos fatores determinantes da judicialização, novas investigações serão conduzidas pelos autores em estudos posteriores.

Em relação ao tipo de ação, 67,2\% (N=125) tiveram apenas o Estado como réu do processo, 2,2\% ( $\mathrm{N}=4)$, apenas o município, e 30,6 ( $\mathrm{N}=57)$, ambos os entes. As 186 ações judiciais deferidas entre os anos de 2004 a 2015 determinaram o fornecimento de 781 medicamentos, dos quais 508 tratamentos foram demandados pelas 125 ações deferidas apenas contra o Estado, outros 28 tratamentos foram determinados pelas quatro ações movidas contra o município e 245 tratamentos compuseram as 57 ações demandadas contra ambos os entes. O caráter exponencial do crescimento das demandas judiciais referendada na literatura ${ }^{9}$ foi também constatado neste trabalho. No ano de 2004, havia apenas quatro ações; em 2015, esse número subiu para 151 processos. Esse dado mostra que os significativos avanços na assistência farmacêutica, tanto do ponto de vista de aumento do financiamento ${ }^{11}$ como da inclusão de novas alternativas terapêuticas na RENAME, foram insuficientes para diminuir o fenômeno da judicialização.

A estimativa do valor necessário para o cumprimento das ações judiciais foi calculada em três cenários diferentes. No caso do cenário A, em que a administração pública teria que fornecer apenas o medicamento de referência para cada um dos tratamentos judicializados, os custos de aquisição somaram valores mais vultosos, uma vez que os custos dos medicamentos de referência são significativamente mais elevados que os genéricos ou os similares equivalentes.

No cenário B, caso as ações fossem deferidas pela DCB, permitindo assim que a administração pública optar pela opção mais vantajosa entre o medicamento de referência e seu correspondente genérico, houve um aumento da concorrência entre os laboratórios farmacêuticos que produzem determinado item, o que implica redução do preço unitário e, consequentemente, otimização dos recursos públicos. Cabe destacar que o processo de compra de medicamentos em sistemas públicos de saúde é realizado por meio de processo licitatório regido pela Lei Federal no 8.666/1993 ${ }^{15}$. No que diz respeito à aquisição de medicamentos, a regulamentação é feita pela Lei $n^{\circ} 9.787 / 1999^{14}$, que determina que as aquisições de medicamentos, sob qualquer modalidade de compra, e as prescrições médicas e odontológicas de medicamentos, no âmbito do SUS, adotarão obrigatoriamente a DCB. Dessa forma, os medicamentos genéricos, quando houverem, terão preferência sobre os demais em condições de igualdade de preço. Entretanto, sobrepondo-se a isso, muitas vezes as ações judiciais são deferidas em razão de determinada marca comercial, fato que incorre, no mínimo, a uma contradição do princípio constitucional, visto que, ao fazê-lo, o judiciário não permite que o poder público adquira a opção mais vantajosa, pois o medicamento de referência em geral é o mais caro.

No cenário C, caso as ações fossem deferidas pela DCB, permitindo a administração pública optar pela opção mais vantajosa entre o medicamento de referência, genérico ou similar intercambiável, foi verificado a maior economia dos recursos públicos.

Ferraz e Vieira ${ }^{11}$, em estudo pioneiro sobre as diferenças de preços entre medicamentos genéricos e de referência no Brasil, demonstraram que os genéricos podem ter preço $40 \%$ menor. Esse fato corrobora a importância dessa classe de medicamentos para que o princípio da economia seja alcançado dentro do SUS.

Um estudo que analisou o impacto financeiro da aquisição de medicamentos por meio de licitação pública, com a exigência da apresentação de testes de biodisponibilidade e/ou bioequivalência para o componente básico da assistência 
farmacêutica, identificou um aumento de mais de 100 vezes do custo quando foram adquiridos medicamentos de referência ou genérico ${ }^{19}$. Embora, no Brasil, estima-se que os genéricos tenham sido introduzidos com preços em média $40 \%$ menores do que os medicamentos de referência ${ }^{18}$ e que a política de medicamentos genéricos seja apontada como forma de redução de preço, isso é verdade na comparação com os medicamentos de referência. Os similares são vendidos no Brasil por preço inferior aos genéricos, principalmente nas licitações públicas, de forma que estes últimos não competem com os primeiros em termos de preço ${ }^{19}$.

Em relação aos custos médios das ações, também se verifica que a aquisição de medicamentos similares, comparados aos genéricos e aos de referência, é a que gera maior economia. Contudo, resta sempre a dúvida sobre a qualidade dos medicamentos, principalmente no que se refere aos similares.

Alguns estudos avaliaram a qualidade dos medicamentos fornecidos pelo SUS e apontam para a baixa qualidade dos produtos similares que são adquiridos por meio de licitação pública ${ }^{32,33}$. Contudo, esses estudos são antigos, e muitas regulamentações foram aprimoradas ao longo dos anos com vistas a aumentar a qualidade dos medicamentos comercializados no Brasi ${ }^{34}$. A exemplo disso, podemos citar a Resolução da Diretoria Colegiada (RDC) $n^{\circ} 134 / 2003$, que estabeleceu critérios para a adequação dos medicamentos similares já registrados e comercializados no Brasil, obrigando os detentores de registro de medicamentos similares a apresentar estudos comparativos com o medicamento de referência, tais como equivalência farmacêutica, perfil de dissolução e bioequivalência/biodisponibilidade relativa $(\mathrm{BD} / \mathrm{BE})$, se aplicável ao fármaco e forma farmacêutica ${ }^{35}$.

A literatura destaca que o registro sanitário no Brasil realiza uma análise voltada à oferta no mercado brasileiro e considera a eficácia e a segurança do medicamento de acordo com a indicação à qual se destina ${ }^{1}$. Dessa forma, nada impediria que a administração pública pudesse se utilizar de todas as opções terapêuticas disponíveis, sejam elas de referência, genéricos e similares, para o cumprimento dessas ações judiciais, bem como para a disponibilização desses fármacos nos diferentes componentes da assistência farmacêutica.

Retomando o princípio da economia e analisando as regras atuais do componente da assistência farmacêutica básica, definidos pela Portaria $n^{\circ} 1.555 / 2013^{30}$, que estabelece o valor investido pelas esferas federal, estadual e municipal de $\mathrm{R} \$ 9,82$ habitante/ano, os valores economizados (ao longo dos 12 anos de estudo) seriam de $\mathrm{R} \$ 839.365,39$ e $\mathrm{R} \$ 1.235 .626,76$ quando comparados o cenário B com o A e o cenário C com A, respectivamente. Essa diferença de custo de aquisição dos medicamentos, conforme relatado anteriormente, representa o orçamento de mais de seis anos, comparando o cenário $\mathrm{B}$ com $\mathrm{A}$, e de quase dez anos, comparando o cenário $\mathrm{C}$ com $\mathrm{A}$, para a aquisição de medicamentos do componente básico da assistência farmacêutica.
Diante desses cenários, se, por um lado, as ações judiciais asseguram o direito à assistência farmacêutica a algumas pessoas, por outro, comprometem o acesso da população geral ao impor ao sistema público gastos excessivos e não condizentes com a realidade financeira destinada à execução das políticas públicas de saúde. Estudos relacionados ao fenômeno da judicialização apontam que a violação da questão orçamentária se reflete em uma redução da oferta a medicamentos, exames e consultas, e inverte a lógica dos serviços integrais de promoção, proteção e recuperação da saúde, desconsiderando os princípios primordiais de universalidade, integralidade e equidade que são o sustentáculo do SUS².

Alguns estudos têm apontado que a judicialização em saúde é uma forma de pressão constante do poder judiciário sobre o poder executivo no sentido da busca de melhorias. Entretanto, não são os gestores políticos, e sim os gestores técnicos os principais penalizados pelos excessos do poder judiciário quando o assunto é a judicialização da saúde. O problema da escassez de recursos é destacado como um dos principais entraves ao avanço da saúde pública no país. Enquanto os gestores políticos alternam-se diante do problema, visto que ocupam cargos livres de nomeação e exoneração, os gestores técnicos terão que lidar constantemente com ele, pois são de provimento efetivo e, assim, deverão encontrar os meios para minimizar os efeitos e assegurar que os demais serviços continuem sendo oferecidos.

Apesar de este estudo retratar apenas uma realidade, há uma grande possibilidade que esse fenômeno esteja acontecendo em outros municípios do país. Dessa forma, este trabalho poderá ser usado como forma de questionamento e avaliação das políticas de acesso aos medicamentos em outros cenários.

Este estudo avaliou os dados da judicialização de medicamentos de um pequeno município no interior do Rio Grande do Sul, porém fez uma análise distinta dos trabalhos já publicados com um olhar sobre o custo da aquisição pelo nome de marca e o impacto que essa restrição da livre concorrência traz para o orçamento público. Em tempo de recursos finitos e necessidades infinitas, a otimização dos recursos financeiros é imprescindível para os gestores públicos. No que se refere aos medicamentos, continua a necessidade da garantia da qualidade dos produtos dentro do menor custo possível. A análise dos resultados pode contribuir para a discussão do binômio qualidade-custo para o SUS e para a judicialização da saúde.

Sugere-se que o poder judiciário considere a escassez de recursos públicos da saúde em seus despachos e zele pelo bem comum. Diante de uma inevitável ação judicial, é indispensável que haja o deferimento por meio da DCB, visto que, assim, há racionalização dos gastos dos recursos públicos.

Com esses resultados, concluímos que as ações judiciais deferidas em razão da marca comercial vão de encontro ao princípio da economia e acabam por impor gastos muito maiores ao sistema público de saúde diante daquelas cumpridas com seus correspondentes genéricos e/ou similares. 


\section{REFERÊNCIAS}

1. Pepe VLE, Figueiredo TA, Simas L, Osorio-de-Castro CGS, Ventura M. A judicialização da saúde e os novos desafios da gestão da assistência farmacêutica. Cien Saude Colet. 2010;15(5):2405-14. PMid:20802873. http://dx.doi.org/10.1590/S1413-81232010000500015.

2. Machado MA, Acurcio FA, Brandão CM, Faleiros DR, Guerra AA Jr, Cherchiglia ML, et al. Judicialization of access to medicines in Minas Gerais state, Southeastern Brazil. Rev Saude Publica. 2011;45(3):590-8. PMid:21445458. http://dx.doi.org/10.1590/S0034-89102011005000015.

3. Chieffi AL, Barata RB. 'Judicialization' of public health policy for distribution of medicines. Cad Saude Publica. 2009;25(8):1839-49. PMid:19649425. http://dx.doi.org/10.1590/S0102-311X2009000800020.

4. Diniz D, Medeiros M, Schwartz IVD. Consequences of the judicialization of health policies: the cost of medicines for mucopolysaccharidosis. Cad Saude Publica. 2012;28(3):479-89. PMid:22415180. http://dx.doi.org/10.1590/ S0102-311X2012000300008.

5. Brasil. Constituição da República Federativa do Brasil de 1988. Diário Oficial da União, Brasília, 5 de outubro de 1988.

6. Brasil. Lei no 8.080 de 19 de setembro de 1990. Dispõe sobre as condições para a promoção, proteção e recuperação da saúde, a organização e o funcionamento dos serviços correspondentes e dá outras providências. Diário Oficial da União, Brasília, 20 de setembro de 1990.

7. Monteiro WM, de Melo GC, Massunari GK, Hübner DV, Tasca RS. Avaliação da disponibilidade de medicamentos genéricos em farmácias e drogarias de Maringá (PR) e comparação de seus preços com os de referência e similares. Rev. Bras. Ciênc. Farm. 2005;41:333-43.

8. Sant'Ana JMB, Pepe VLE, Figueiredo TA, Osorio-de-Castro CGS, Ventura M. Rational therapeutics: health-related elements in lawsuits demanding medicines. Rev Saude Publica. 2011;45(4):714-21. PMid:21739079. http:// dx.doi.org/10.1590/S0034-89102011005000042.

9. Figueiredo TA, Pepe VLE, Osorio-de-Castro CGS. A sanitary focus on medicines lawsuit. Physis Rev. Saúde Coletiva. 2010;20:101-18.

10. Vargas-Peláez CM, Rover MRM, Leite SN, Rossi Buenaventura F, Farias MR. Right to health, essential medicines, and lawsuits for access to medicines: a scoping study. Soc Sci Med. 2014;121:48-55. PMid:25306409. http://dx.doi. org/10.1016/j.socscimed.2014.08.042.

11. Ferraz OLM, Vieira FS. The right to health, scarce resources, and equity: inherent risks in the predominant legal interpretation. Dados. 2009; 52(1):223-51. http://dx.doi.org/10.1590/S0011-52582009000100007.

12. Mello AF, Soares LSS, Areda CA, Blatt RC, Galato D. Uma abordagem econômica de processos judiciais de medicamentos impetrados contra um município do sul do Brasil. J. Bras. Econ. Saúde. 2016;1:39-46.

13. Vieira FS, Zucchi P. Distortions to national drug policy caused by lawsuits in Brazil. Rev Saude Publica. 2007;41(2):214-22. PMid:17384795. http:// dx.doi.org/10.1590/S0034-89102007000200007.

14. Brasil. Lei no 9.787 de 10 de fevereiro de 1999. Altera a Lei no 6.360, de 26 de setembro de 1976, que dispõe sobre a vigilância sanitária, estabelece o medicamento genérico, dispõe sobre a utilização dos nomes genéricos em produtos farmacêuticos e dá outras providências. Diário Oficial da União, Brasília, 11 de fevereiro de 1999.

15. Brasil. Lei no 8.666, de 21 de junho de 1993. Regulamenta o art. 37, inciso XXI, da Constituição Federal, institui normas para licitações e contratos da
Administração Pública e dá outras providências. Diário Oficial da União, Brasília, 22 de junho de 1993.

16. Dias CRC, Romano-Lieber NS. Processo da implantação da política de medicamentos genéricos no Brasil. Cad Saude Publica. 2006;22(8):1661-9. PMid:16832537. http://dx.doi.org/10.1590/S0102-311X2006000800014.

17. Bermudez J. Generic drugs: an alternative for the Brazilian market. Cad Saude Publica. 1994;10(3):368-78. PMid:14762545. http://dx.doi.org/10.1590/ S0102-311X1994000300016.

18. Vieira FS, Zucchi P. Price differences between generic and innovator medicines in Brazil. Rev Saude Publica. 2006;40(3):444-9. PMid:16810368. http://dx.doi.org/10.1590/S0034-89102006000300012.

19. Bevilacqua G, Farias MR, Blatt CR. Procurement of generic medicines in a medium size municipality. Rev Saude Publica. 2011;45(3):583-9. PMid:21503556. http://dx.doi.org/10.1590/S0034-89102011005000020.

20. Nishijima M. Os preços dos medicamentos de referência após a entrada dos medicamentos genéricos no mercado farmacêutico brasileiro. Rev Bras Econ. 2008;62(2):189-206. http://dx.doi.org/10.1590/S0034-71402008000200004.

21. Miranda ES, Pinto CB, Reis AL, Emmerick IC, Campos MR, Luiza VL, et al. Availability of generic drugs in the public sector and prices in the private sector in different regions of Brazil. Cad Saude Publica. 2009;25(10):214758. PMid:19851615. http://dx.doi.org/10.1590/S0102-311X2009001000006.

22. Silvério MS, Leite ICG. Quality of prescriptions in a city of Minas Gerais: a pharmacoepidemiological approach. Rev Assoc Med Bras. 2010;56(6):67580. PMid:21271134.

23. Barreto JL, Pereira MT, Guimarães MCL, Penaforte TR, Formigli VLA. Perfil das demandas judiciais por medicamentos em municípios do Estado da Bahia. Rev. Baiana Saúde Pública. 2014;37:536-52.

24. Rio Grande do Sul. Tribunal de Justiça [Internet]. 2017. [citado em 2017 jan 24]. Disponível em: http://www.tjrs.jus.br/busca/?tb=proc

25. Brasil. Ministério da Saúde. Secretaria de Ciência, Tecnologia e Insumos Estratégicos. Departamento de Assistência Farmacêutica e Insumos Estratégicos. Relação Nacional de Medicamentos Essenciais: Rename. 9. Ed. Brasília.

26. Agência Nacional de Vigilância Sanitária - ANVISA [Internet]. 2017. [citado em 2017 jan 24]. Disponível em: http://portal.anvisa.gov.br

27. Costa RDF, Osorio-de-Castro CG, Silva RM, Maia AA, Ramos MC, Caetano R. Aquisição de medicamentos para a Doença de Alzheimer no Brasil: uma análise no sistema federal de compras, 2008 a 2013. Cien Saude Colet. 2015;20(12):3827-38. PMid:26691807.

28. Calculador [Internet]. 2017. [citado em 2017 jan 24]. Disponível em: http:// www.calculador.com.br/tabela/indice/IPCA

29. Brasil. Lei no 10.742 de 6 de outubro de 2003. Define as normas de regulação para o setor farmacêutico e dá outras providêcias. Diário Oficial da União, Brasília, 7 de outubro de 2003.

30. Brasil. Portaria $\mathrm{GM} \mathrm{n}^{\circ} 1.555$ de 30 de julho de 2013. Dispõe sobre as normas de financiamento e de execução do Componente Básico da Assistência Farmacêutica no âmbito do Sistema Único de Saúde (SUS). Diário Oficial da União, Brasília, 31 de julho de 2013.

31. Soares L, Diehl EE, Leite SN, Farias MR. A model for drug dispensing service based on the care process in the Brazilian health system. Braz 
J Pharm Sci. 2013;49(1):107-16. http://dx.doi.org/10.1590/S198482502013000100012

32. Batistic MA, Auricchio MT, Markman BEO. Avaliaçäo da qualidade de comprimidos revestidos e soluçöes orais de sulfato ferroso utilizados no tratamento da anemia ferropriva. Rev Inst Adolfo Lutz. 1998;57:25-8.

33. Bianchin MD, Blatt CR, Soares A, Külkamp-Guerreiro IC. Evaluation of the quality of propranolol and enalapril tablets supplied in the public health system in a southern Brazilian city. Cien Saude Colet. 2012;17(2):491-8. PMid:22267043. http://dx.doi.org/10.1590/S1413-81232012000200022.
34. Rumel D, Nishioka S, Santos AA. Drug interchangeability: clinical approach and consumer's point of view. Rev Saude Publica. 2006;40(5):921-7. PMid:17301916. http://dx.doi.org/10.1590/S0034-89102006000600024.

35. Brasil. RDC no 134, de 29 de maio de 2003. Dispõe sobre a adequação dos medicamentos já registrados. Diário Oficial da União, Brasília, 30 de maio de 2003.

Recebido em: Jan. 24, 2017 Aprovado em: Abr. 06, 2017 